\title{
Automatic Search and Analysis on Sinusoidal Whistle Signal of Captive Bottlenose Dolphins
}

\author{
ShengChen ${ }^{1}$ Fuqiangniu ${ }^{1}$ Weiliu $^{2}$ \\ (1. Third Institute Of Oceanograph,State Oceanic Administration ) \\ (2. The Reserve Management Office of Chinese White Dolphins and Amphioxus in Xiamen) \\ Xiamen, Fujian, P.R.China \\ 361000 Sheng_c@tio.org.cn
}

\begin{abstract}
$^{1}$
It is inefficient to observe the biological behavior of bottlenose dolphin full time artificially. A kind of correlation method was proposed, the acoustic spectrum of Sinusoidal whistle signals emitted from captive dolphins from 19:00 to 08:00 in a year were searched automatically. The results show that the occurrences of Sinusoidal signals were relatively high in August, September and October. There was the highest proportion in
\end{abstract} August. By comparison and analysis with a year's feeding records on dolphin's behaviors, water temperature and salinity, it can be found that when the frequency of occurrence for this type signals was larger, the dolphins had more bad mood symptoms. That would be related with the water temperature and water salinity. The breeder can solve the problem by changing water or adjusting the food structure as so on. The results may be valuable for further study on dolphin biological behavior and animal protection.

Key words: Bottlenose dolphin; Sinusoidal whistle signal; Acoustic spectrum

\section{INTRODUCTION}

Bottlenose dolphin is also called Tursiops truncatus,. It is one of the dolphins of cetaceans species. Bottlenose dolphins are mainly in shallow waters near land, widely distributed in the sea of temperate and tropical oceans. Dolphins have "underwater sonar", it can acquire sound signals through its sonar system.

At present, both here and abroad, dolphin sounds are generally classified into three categories[1,2]:, click, whistles and burst pulses. Dolphin's whistles signal is a continuous time signal. The duration varies from several hundred milliseconds

\footnotetext{
1 This work was supported by the Scientific Research Foundation of Third Institute of Oceanography, SOA(NO.2015026, No. 2017031)
}

to several seconds. The frequency distribution is usually between thousands of Hertz and tens of thousands of Hertz. It is mainly used for dolphin individuals or groups to communicate with each other, emotional expression and information transmission [3]. Studying the whistles of dolphins is of great significance to understand their biological behavior.With the development of bioacoustics, the research on the characteristics of biological acoustic signals has attracted wide attention.By collecting and analyzing the whistles of a large number of dolphins, Driscoll[4] and Bazua-Duran[5] divided the dolphin whistles into 6 categories according to the track shape of the frequency change in the time frequency diagram.(1) Constant frequency: (2) Upsweep: (3) Downsweep; (4) Concave or valley; (5) Convex or hill; (6) Sinusoidal.

Wang Ding, Bernd W, et.al. compared the whistles among seven odontocete species[6]. Wei Chong, Xu Xiao mei, et.al. study on whistle characteristics of captive bottlenose dolphins during swimming and under training. It was found that under the training state, there were a lot of scavenging signals and there were more Sinusoidal whistle signals in free styles[7]. In this paper, we want to focus on the analysis of the basic acoustic characteristics of the bottlenose dolphins in the captive pool .That would not be disturbed by the training. Therefore, we choose the Sinusoidal whistle signal as the research object. The track shape of the Sinusoidal whistle signal is similar to the sinusoidal signal, and the spectrum shape of the signal is descended after the rise and then undulating forward (or first descending and then rising), of which at least two inflection points appear.

In this paper, the whistle signals were collected from the captive bottlenose dolphins. The Sinusoidal whistle signals in one year were picked out automatically. The track shape of the Sinusoidal whistle signal is similar to the sinusoidal signal, the spectrum shape of the signal is descended after the rise and then undulating forward (or first descending and then rising), of which at least two inflection points appear. As shown in Fig.1. Some of are shaped like letters "M", shown in fig (a), some of them are shaped like letters "N", shown in fig (b), some of them are shaped like letters "W", shown in fig(c); some of them are continuous inflection points, shown in fig (d).

In this paper, the behaviors of the dolphins were also analyzed, water temperature and water salinity in the corresponding environment were totaled. Some conclusions will provide important reference materials for future related 
research and lay the foundation for better protection of dolphins.

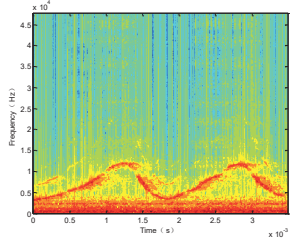

(a)'M’type

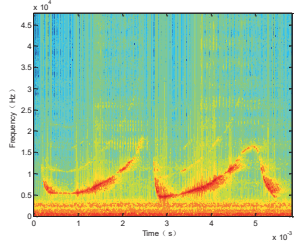

(c)'W'type

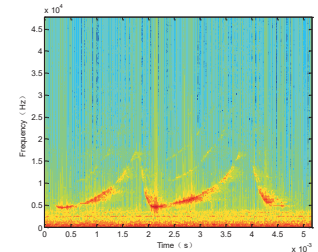

(b)'N'type

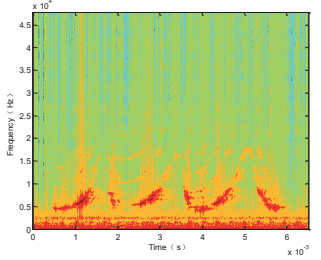

(d)'continues W'type
Fig. 1 the recorded sinusoidal signal

\section{RECORDING THE SIGNALS}

\section{A experimental dolphins}

From March 2017 to April 2018, the sound of two bottlenose dolphins in Xiamen dolphin protected island was recorded in the whole day. The recording time per hour was $5 \mathrm{~min}, 1$ days and 24 hours, and a total of 12 months of original sound data were recorded. Two bottlenose dolphins were adult male (M1) and adult male (Y2), respectively. The basic information of the dolphins during the experiment is shown in Table 1.

TABLE 1

The basic situation of two bottlenose dolphin

$\begin{array}{llll}\text { body } & \text { weight } & \begin{array}{l}\text { Age } \\ \text { estimation }\end{array} & \text { Mature } \\ \text { length } & & \text { state } & \text { Captivity pool }\end{array}$

$\begin{array}{llllll}240 \mathrm{~cm} & 134 \mathrm{~kg} & 23 & & \text { Adult } & 2 \# \\ 213 \mathrm{~cm} & 120 \mathrm{~kg} & 19 & \text { adult } & 2 \#\end{array}$

\section{$B$ The construction of experimental system}

The two bottlenose dolphins are in captivity in the $2 \#$ pool of Xiamen's Fire Island. The diameter of the pool is 5 meters, and the depth is 5 meters. The experimental system and experimental scene are shown in Fig.2.

The sound recorder used in this experiment is $\mathrm{SM}++$ in France with a omnidirectional hydrophone. The sensitivity of the hydrophone is $25 \mathrm{~Hz}-45 \mathrm{kHz}( \pm 3 \mathrm{~dB})$, and the sensitivity is - $165 \pm 3 \mathrm{~dB}$ re: $1 \mathrm{~V} / \mu \mathrm{Pa}$; the sampling rate is $96 \mathrm{kHz}$, the conversion number is 16 bit full spectrum two channels; it records 5 minutes or 10 minutes per hour; the preamplifier gain is set up $6 \mathrm{~dB}$, the range is $\pm 1 \mathrm{VDC}$. The total capacity is $512 \mathrm{~GB}$, the storage time is 3 months.

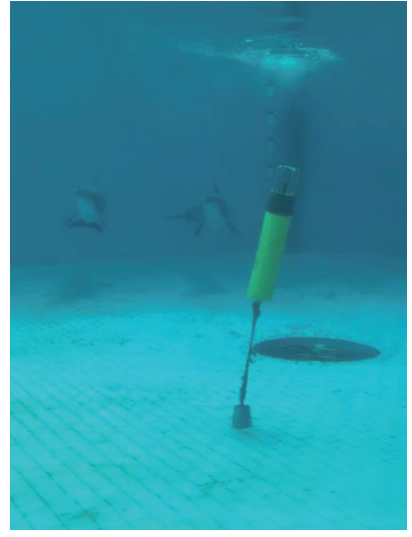

Fig2. experimental scene

\section{DATA PROCESSING METHOD}

In the case of marine mammals, a number of research groups are now regularly deploying autonomous recorders which collect data over many months, thereby it is inefficient to observe the biological behavior of bottlenose dolphin full timer artificially. Many methods of automatically detecting and classifying tonal sounds are proposed, including Short Time energy method, Time Frequency(TF) correlation method, Gauss mixture model method and Frequency contour tracing method[8-12] and so on. In this paper, we address Time Frequency correlation method. First, the echo signal is mapped into the $\mathrm{TF}$ domain by the RID(Reduced Interference Distribution). Next, the RID-SV(singular value) feature is extracted according to the SVD(singular value decomposition) on the TF domain. Finally, the correlation method is used as the classify to choose the Sinusoidal whistle signals.

\section{A Reduced Interference Distribution(RID)}

The analysis technology of TF is a tool to describe the energy distribution in both the time domain and frequency domain. In the TF space, the time-varied frequency spectrum can be observed easily. The WVD(Wigner-Ville Distributio) is a kind of effective TF tool and is suitable to analyze a multi-component signal like a bio-dolphin click. The WVD is a fundamental part of the Cohen's class TF distributions, whereas its performance is limited seriously by the interference of the cross-term which is produced by the interaction between the signal components. The cross-term interference often creates an echo information distortion which leads to erroneous classification. A number of researchers has investigated various kernels for reducing this interference. The RID is designed by the first kind Bessel function of order one [13]. This distribution can be described as:

$$
\begin{aligned}
& R I D(t, \omega)=\int_{\mu} e^{-j \omega \tau}\left[\int_{\mu} \frac{2}{\pi \alpha|\tau|} \sqrt{1-\left(\frac{\mu-t}{\alpha \tau}\right)^{2}}\right] \\
& \left.\times \Pi\left(\frac{\mu-t}{2 \alpha \tau}\right) f\left(\mu+\frac{\tau}{2}\right) f^{*}\left(\mu-\frac{\tau}{2}\right) d \mu\right] d \tau
\end{aligned}
$$

Where:

$$
\Pi\left(\frac{\mu-t}{2 \alpha \tau}\right)=\left\{\begin{array}{cc}
1 & |\mu-t| \leq \alpha|\tau| \\
0 & \text { otherwise }
\end{array}\right.
$$


Because of the low-pass filter property of the Bessel kernel, the RID can not only suppresses the interference of cross-term effectively in the time-frequency space, it also sustains high resolution[14].

\section{$B$ Singular Value Decomposition}

Based on the theory of SVD, A two-dimension matrix "A" can be decomposed into a series of sub-unitary matrix corresponding to singular value vector, and this process is described in the following equation:

$$
\left\{\begin{array}{c}
A=U \Lambda V^{H} \\
\Lambda=\operatorname{diag}\left\{\lambda_{1}, \ldots, \lambda_{i}, \ldots, \lambda_{k}, 0 \ldots 0\right\} \\
K=\operatorname{rank}(A)=\min (m, n)
\end{array}\right.
$$

The singular value matrix" $\Lambda$ " is unique corresponding to a different matrix, even though generally the matrix "U" and "V" is variable. The SVD not only has many very good properties, such as shifting invariance, rotating invariance and scaling invariance, but also it can also remain stable in the noise environment. Therefore, the SVD has the ability to extract the inherent property of the arbitrary matrix both steadily and effectively. Furthermore, it is not to be neglected that the feature vector can be compressed to the benefit of reducing the complexity of algorithm.

\section{$B$ cross-correlation process}

First of all, a two dimensional kernel is established according to the known signal function。Given the kernel $\Lambda_{\mathrm{k}}(\mathrm{t}, \mathrm{f})$ and the spectrogram, the output of the cross-correlation process for each frame of the spectrogram is given by

$$
\alpha(t, f)=\sum_{t_{0}} \sum_{f} \Lambda k\left(t_{0}, f\right) \Lambda S\left(t-t_{0}, f\right)
$$

where $\Lambda \mathrm{S}(\mathrm{t}, \mathrm{f})$ is the spectrogram, and the limits of summation are specified by the size of $\mathrm{k}$ in time and frequency. Note that the correlation is in the time and frequency dimension, as would be used for two-dimensional cross-correlation. The result of the cross-correlation operations is a time and frequency series with a "recognition score" $\alpha(\mathrm{t}, \mathrm{f})$ for each point in time and frequency, i.e.,a value representing the closeness of match between spectrogram and the kernel.

\section{CLASSIFICATION RESULTS AND ANALYSIS}

\section{A. Classification Results}

After a number of classification tests by above methods, the results are shown in Table 2. The actual manual selection results are shown in Table 2 too for comparison.

Table 2. the comparison of related method selection and manual selection

Experiment Test signal Correct Correct rate

\begin{tabular}{cccc}
\hline times & number & number & \\
\hline 1 & 15 & 13 & $87 \%$ \\
2 & 20 & 17 & $85 \%$ \\
3 & 50 & 46 & $92 \%$ \\
4 & 25 & 21 & $84 \%$ \\
5 & 40 & 36 & $90 \%$ \\
Total & 150 & 133 & $89 \%$ \\
\hline
\end{tabular}

It can be seen from table 2 , that the correct rate of each test is greater than or equal to $85 \%$ when the Sinusoidal whistle signal is classified by this method. This indicates that it is feasible to select out the Sinusoidal whistle signal which recorded in the experiment by this method.

The above method is used to classify the sound signals of dolphins in the pool recorded in one year. The statistics distribution of Sinusoidal whistle signals is shown in Fig5..

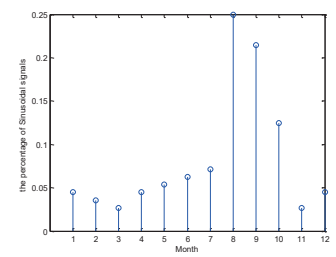

(a)

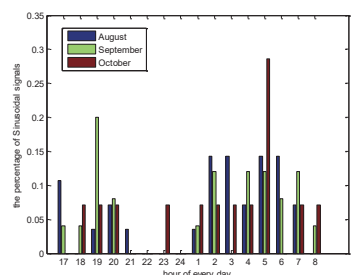

(b)
Fig5.(a) the percentage of Sinusoidal whistle signal in a year; (b) the daily distribution of Sinusoidal whistle signal within three months.

It can be seen from Fig5.(a) that the occurrence of Sinusoidal whistle signal is relatively high in August, September and October. There were the highest proportion in August. According to the records of dolphin behavior within one year, the two dolphins in one year were often found to had bad mood symptoms, such as irritability, repeated butt and pat pool wall, violent impact on glass window, did not cooperate with training as so on. The frequencies of the occurrence of bad mood were recorded. After totaling, the distribution of the amount of bad moods was shown as fig6..

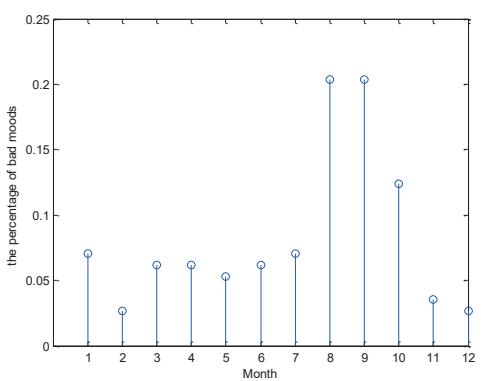

Fig6. the proportion of bad moods of dolphins in one year As you can see from the Fig6., the dolphins in the 
pool had the higher percentage of bad moods in August, September and October, which months coincides with the higher percentage of Sinusoidal whistle signals. As shown in the Fig.5(a).The proportion of Sinusoidal whistle signal and bad moods monthly are draw on the same picture, shown in the Fig.7.

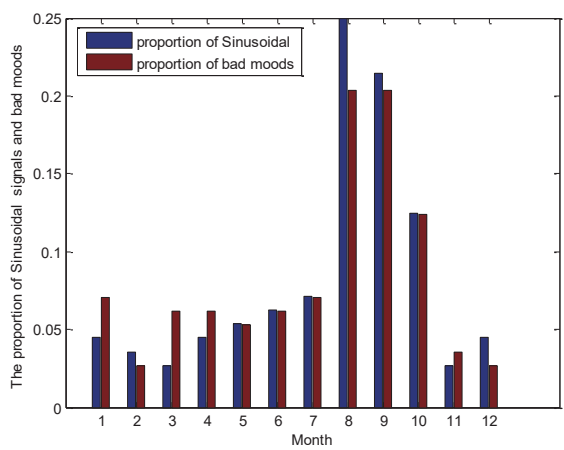

Fig7. the proportion of Sinusoidal whistle signal and bad moods

As can be seen from the fig7., the peak period of dolphins issuing sinusoidal signals is often accompanied by bad moods and uncoordinated training symptoms. It is presumed that the phenomenon may be related to the environment. The environment including the pool water temperature and salinity were observed and calculated. The result of the monthly average temperature and salinity in a year are shown in the Fig8..

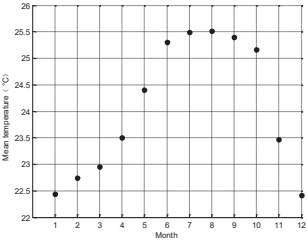

(a)

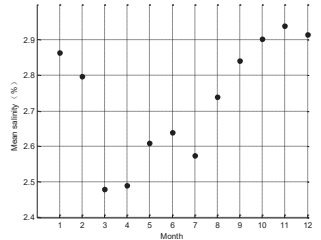

(b)
Fig8. (a) monthly mean temperature; (b) monthly mean salinity

From the Fig8.(a), the average temperature from June to October is higher, more than $25^{\circ} \mathrm{C}$, and the average temperature in August is the highest, reaching $25.5^{\circ}$ C. From fig8. (b), the average salinity of August to October is higher than 2.7\%.It can also found in the Fig5. (b), the distribution of sinusoidal signals from August to October is also in the period of higher temperature in a day. It can be speculated that when the water temperature and salinity are higher, the dolphins emitted more sinusoidal signals.

By lowering the water temperature and regulating the salinity of food, the feeding staff found that the sinusoidal signal from dolphins actually decreased. It proofed that the higher water temperature and salinity would stimulate the dolphins to emit more sinusoidal signal. The breeder can solve the problem by changing water or adjusting the food structure as so on

\section{CONCLUSION}

According to the above analysis, we can know: 1.by above method, the Sinusoidal whistle signal can be searched automatically, the correct rate is more than $80 \% ; 2$. when the water temperature in the pool is over $25^{\circ} \mathrm{C}$,both salinity exceeds $2.7 \%$, the captive dolphins will emit more Sinusoidal whistle signals, At the same time, it shows bad moods ;3. The breeder can improve the dolphin moods by changing water or adjusting the food structure. In the future work, we can further study the sound of dolphins in different sea areas in the natural environment, which will be of great significance to understanding and mastering marine life and marine environment.

\section{REFERENCES}

[1] Whitlow W L Au.The Sonar of dolphins[M].New York:Springer-Verlag,1993:119-134.

[2] Whitlow W L Au. The Sonar of dolphins. New York: Springer-Verlag, 1993;

[3] Herzing D L. Acoustics and social behavior of wild dolphins implications for a sound society. New York: Hearing By Whales and Dolphins, 2000: 225-272;

[4] Wang Ding, Bernd W, Evans W E. Comparisons of whistles among seven odontocete species. In: Sensory Systems of Aquatic Mammals, Kastelein R A, Thomas J A, Nachtigall P E Eds., De Spil Publishers Woerdem, Netherlands, 1995: 299-323

[5] Driscoll A D. The whistles of Hawaiian spinner dolphins(Stenella longirostris). Master's thesis, University of California Santa Cruz, 1995;

[6] BazÚa-Duran C. Comparisons of whistles among differ-ent populations of dolphins (Tursiops truncatus) from the Gulf of Mexico. Master's thesis, Universidad National Autonoma De Mexico, Mexico, DF Mexico, 1997;

[7] WEI Chong XU Xiaomei ZHANG Yu,et.al.Comparison study on whistle characteristics of captive bottlenose dolphins(Tursiops truncatus) during swimming and under training ACTA ACUSTICA 2014,39(7):452-458;

[8] Mellinger D K, Martin S W.Morrissey S W,et al. A method for detecting whistles, moans and other frequency contour sounds. Journal of the Acoustical Society of America. 2011.129(6):4055--4061.

[9] Parada P P, Cardenal-Lopez A.Using Gaussian mixture models to detect and classify dolphin whistles and pulses. Journal of the Acoustical Society of America ,2014,135(6):3371-3380.

[10] Lin $\mathrm{T} \mathrm{H}$, Yu H Y.Chen $\mathrm{C}$ F,et al.Automatic detection and classification of cetacean tonal sounds from a long-term marine observatory.In : Proceedings of Symposium on Underwater Technology.Tokyo: IEEE Press, 2013,1-6.

[11] Gillespie D,Caillat M, Gordon J, et al. Automatic detection and classification of odontocete Whistles. Journal of the Acoustical Society of America,2013 .134(3):2427--2437.

[12] H. H. Ou, W. W. 1. Au, and V. L. Syrmos, "Underwater Ordnance Classifcation Using Time-Frequency Signatures of Backscattering Signals," Oceans 2010,2010.

[13] Z. Guo,L. G. Durand,and H. C. Lee, "The time-frequency distributions of nonstationary signals based on a Bessel kernel," Signal Processing, IEEE Transactions on, vol. 42,pp.1700-1707,1994 